\title{
Proposal for Applicability of Neutrosophic Set Theory in Medical Al
}

\author{
A.Q.Ansari \\ Department of Electrical \\ Engineering \\ Jamia Millia Islamia \\ New Delhi-110025 \\ India
}

\author{
Ranjit Biswas \\ Department of Computer \\ Science Engineering \\ ITM University \\ HUDA Sector 23-A, \\ Gurgaon-122017, Haryana, \\ India
}

\author{
Swati Aggarwal \\ Department of Computer \\ Science Engineering \\ ITM University \\ HUDA Sector 23-A, \\ Gurgaon-122017, Haryana, \\ India
}

\begin{abstract}
Soft computing is an enriching domain that helps to encode uncertainty and imprecision that exists in real world. Integration of soft computing techniques in the systems lends added advantage to the existing sy stems to allow solutions to otherwise unsolvable problems. Fuzzy architecture has been extensively researched and applied in medical domain. This paper suggests incorporating a new logic: Neutrosophic logic in medical domain and also discusses the possibility of extending the capabilities of the fuzzy systems by employing neutrosophic systems.
\end{abstract}

\section{General Terms \\ Medical AI}

\section{Keywords}

Neutrosophic logic, medical AI

\section{INTRODUCTION}

Healthcare industry has been trying to complement the services offered by conventional clinical decision making systems with the integration of fuzzy logic techniques in them [1], [2], [3], [4], [5], [6], [7], [8], [9], [10], [11], [12], [13], [14], [15], [16]. As it is not an easy task for a clinician to derive a fool proof diagnosis so it is advantageous to automate few initial steps of diagnosis which would not require intervention from an expert doctor.

Medical knowledge base is vast, complex and ever changing, it is suggested in the paper to experiment medical databases with the new entrant in the soft computing domain : Neutrosophic logic; which is a generalized logic and possesses all attributes necessary to encode medical knowledge base and capture medical inputs.

Evidence based health care system is discussed here. Clinical reasoning and decision making are phased. Following steps summarize the complete process of diagnosis and suggestive treatment:-
a. General clinical evaluation (History and physical examination)
b. Precise laboratory tests
c. Integration of clinical findings and test results
d. Comparative benefits and risks for alternative courses of action

e. Patients preference (cost of treatment, compliance expectation, drug sensitive)

f. Final therapeutic plan is developed

Considering the above, it is understandable that no fixed treatment strategy comprising specific drug usage and other modalities of treatment can be fixed even for patients of similar nature problems. Moreover scope of ambiguities, uncertainties and indecisiveness in inputs to medical expert system do vary like history (patient's description of the diseased condition), physical examination (especially in cases of uncooperative or less intelligent patients), laboratory-tests (faulty methods or equipments)

As medical diagnosis demands large amount of information processing, large portion of which is quantifiable, also intuitive thought process involve rapid unconscious data processing and combines available information by law of average, so the whole process offers low intra and inter person consistency.

So contradictions, inconsistency, indeterminacy and fuzziness should be accepted as unavoidable as it is integrated in the behavior of biological systems as well as in their characterization. To model an expert doctor it is imperative that it should not disallow uncertainty as it would be then inapt to capture fuzzy or incomplete knowledge that might lead to the danger of fallacies due to misplaced precision.

Considering the above facts, it is suggested in this paper to use Neutrosophic logic for representing medical knowled ge. Section 2 gives the need and basics of neutrosophic logic required for understanding of its working and representation techniques. Section 3 gives the proposal of applying neutrosophic logic to medical domain and lists the advantages of neutrosophic logic. Section 4 gives the future directions and conclusions.

\section{BASICS AND NEED OF NEUTROSOPHIC LOGIC}

\subsection{Fuzzy logic and its limitations}

As the real world data and executions are ambiguous and vague, it was rightly understood by Prof. Zadeh and his pioneered work formalized data representation by introducing fuzzy logic [17]. Fuzzy logic permits the gradual numerical degree of membership that ranges between 0 and 1; contrary to binary values permissible in classical logic. 


\section{Definition 1:}

Let $\mathrm{U}$ denote a universe of discourse. Then a fuzzy set $\mathrm{A}$ in $\mathrm{U}$ is defined as a set of ordered pairs

$$
A=\left\{\left\langle x, \mu_{A}(x)\right\rangle \mid x \in U\right\}
$$

where $\mu_{A}: U \rightarrow[0,1]$ is a function of A that delivers the grade of membership of $\mathrm{x}$ in $\mathrm{A}$ [17].

The notion of using fuzzy logic for inference is much closer to natural inference as we as human beings tend to conclude or judge in uncertain environments where availability of $100 \%$ complete or true information is very rare. Though initially fuzzy logic witnessed slow start and acceptance but gradually it triggered extensive research in soft knowledge processing. It has found applications in domains like classification, pattern recognition, medicine, ecommerce and many more [18], [19],[20],[21]. As this paper deals with medical domain; relevant application of fuzzy logic in this domain can be found in[1],[2],[3],[4],[5],[6],[7],[8],[9],[10],[11],[12],[13],[14],[15],[1 $6]$ and many more.

Table 1 gives the comparative analysis of classical inference systems, fuzzy inference systems and possible futuristic inference systems.

Table 1: Comparative analysis of classical, fuzzy and futuristic inference system

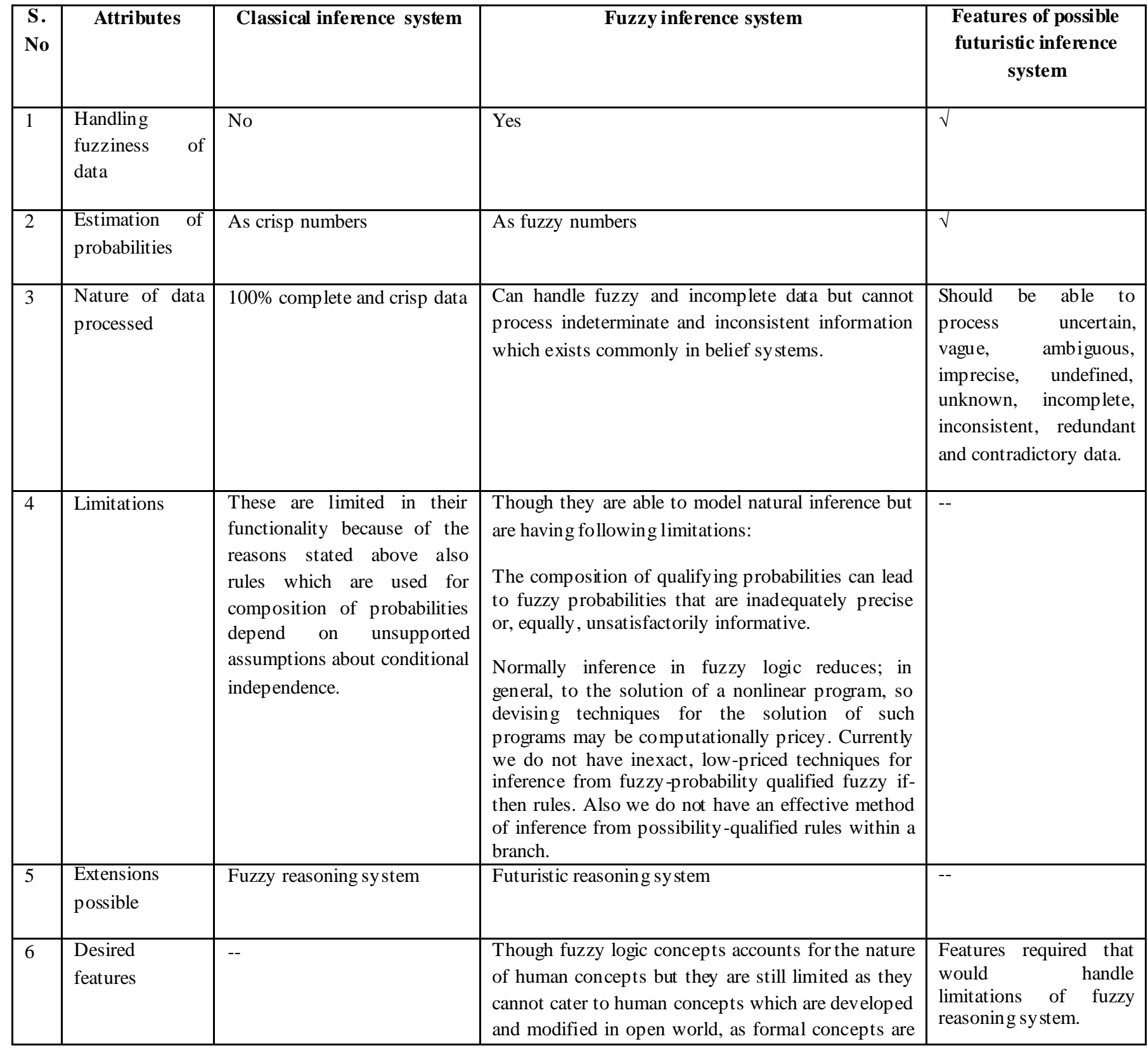




\begin{tabular}{|c|c|c|c|c|}
\hline & & & $\begin{array}{l}\text { fixed in closed world. So it is quite natural that for } \\
\text { open domain problems like open world of human } \\
\text { fuzzy concepts, rigidities of fuzzy formal approach } \\
\text { be further relaxed. } \\
\text { Extension required in the feature set that would } \\
\text { eventually evolve fuzzy reasoning systems to } \\
\text { futuristic reasoning systems }\end{array}$ & \\
\hline 7 & $\begin{array}{l}\text { Current } \\
\text { acceptance / } \\
\text { simulation of } \\
\text { real world } \\
\text { executions }\end{array}$ & Rarely used / Limited & High acceptance/Possible & $\begin{array}{l}\text { Once designed, should } \\
\text { lead to highest } \\
\text { acceptance / } 100 \% \\
\text { support for simulating } \\
\text { real world executions }\end{array}$ \\
\hline
\end{tabular}

Evaluating the points discussed in table 1, it is clear that though fuzzy logic inference systems have desired features that are essential to model natural inference process but still their feature set needs an extension that would help them to extend their capabilities and overcome the present limitations. Current fuzzy inference systems should allow more flexibility in the form of representing paradoxes as it is an important aspect of human reasoning model; as experimentation with the suggested futuristic inference model would extend the understanding and will help to resolve them.

\subsection{Neutrosophic logic}

Quite recently, Neutrosophic Logic has been proposed by Florentine Smarandache which is based on non-standard analysis that was given by Abraham Robinson in 1960s. Neutrosophic Logic was developed to represent mathematical model of uncertainty, vagueness, ambiguity, imprecision, undefined, unknown, incompleteness, inconsistency, redundancy, contradiction [22],[23].

As this paper discusses the possibility of utilizing neutrosophic logic in medical domain so all the representational parameters stated above are very integral to human thinking as well as aptly suits the requirements of medical data representation. It is very rare that a doctor tends to diagnose/ judge the disease in definite environments, imprecision could be due to the lack of confidence on the part of patients in reporting symptoms, or imperfection leads to a doubt about the value of a variable, a decision to be taken or a conclusion to be drawn for the actual symptom. Multiple factors could lead to uncertainty like incomplete knowled ge (ignorance of the patient, limited view on a system because of its complexity), stochasticity (the case of intrinsic imperfection where a typical and single value does not exist), or the acquisition errors (intrinsically imperfect lab observations, the quantitative errors in measures).

So the developed system would have unknown features and behaviors associated, and there would always be unanticipated happening conditions which are uncontrollable - we mean the indeterminacy plays a role as well; a better approach would be using Neutrosophic logic as discussed.

\section{Definition 2:}

In general if $X$ be a universe of discourse, and $M$ a neutrosophic set included in $X$. An element $x$ from $X$ is noted with respect to the set $M$ as $x(T, I, F)$ and belongs to $M$ in the following way:

it is $t \%$ true in the set, $i \%$ indeterminate (unknown if it is) in the set, and $f \%$ false, where $t$ varies in $T, i$ varies in $I, f$ varies in $F$, where Then $T, I, F$ are called neutrosophic components [23].

$T, I, F$ can be standard or non-standard real subsets of ] $-0,1+[$, with sup $\mathrm{T}=\mathrm{t} \_$sup, inf $\mathrm{T}=\mathrm{t} \_$inf,

$\sup I=i \_s u p, \inf I=i \_i n f$,

$\sup F=f \_s u p, \inf F=f \_i n f$,

and $n \_$sup $=\mathrm{t} \_$sup $+\mathrm{i} \_$sup $+\mathrm{f} \_$sup,

$\mathrm{n} \_$inf $=\mathrm{t} \_$inf $+\mathrm{i} \_$inf $+\mathrm{f} \_$inf.

Neutrosophic logic in comparison to other logics, is not confined to range of $[0,1] . T, I, F$ can be assigned over boiling values $(>1)$ or under dried values $(<0)$, but here for practical purposes and to keep the discussion relatively simpler we are assuming the range of $[0,1][23]$.

So, if X (universe of discourse: UOD) is a collection of objects denoted generically by $\mathrm{x}$, then a neutrosophic set $\mathrm{M}$ in $\mathrm{X}$ is characterized by three membership functions: TM truth membership function, IM indeterminacy membership function and FM falsity membership function.

$$
\begin{aligned}
& M\left(T_{M}, I_{M}, F_{M}\right) \\
& \text { and } M=\left\{\left(x, T_{M}(x), I_{M}(x), F_{M}(x) \mid x \in X\right\}\right. \\
& \left.T_{M}(x) \rightarrow\right\rfloor 0,1^{+} \mid \\
& \left.I_{M}(x) \rightarrow\right\rfloor 0,1^{+} \mid \\
& \left.F_{M}(x) \rightarrow\right\rfloor 0,1^{+}
\end{aligned}
$$




$$
\begin{aligned}
& \text { There is no restriction on the sum } \\
& \text { of } T_{M}(x), I_{M}(x) a n d F_{M}(x) \\
& \text { so }^{-} 0 \leq \sup \left(T_{M}(x)\right)+\sup \left(I_{M}(x)\right)+\sup \left(F_{M}(x)\right) \leq 3^{+} \text {. }
\end{aligned}
$$

\subsection{Reasons for extending fuzzy logic}

To summarize following reasons are the major driving force for extending fuzzy logic:

i. Because a paradox, as proposition, cannot be described in fuzzy logic.

For example a paradox is a proposition which is true and false in the same time, Neutrosophic logic representation for the same would be NL (paradox) $=(1, i, 1)$, but this notation is not applicable to fuzzy logic, because if FL(paradox) $=1$ (the truth) then automatically the fuzzy component of falsity is 0 . That's why neutrosophics is fascinating to study.

ii. Neutrosophic logic clearly distinguishes between 'relative truth' and an 'absolute truth', while fuzzy logic does not.

Basically, neutrosophic logic is a generalization of fuzzy logic based on neutrosophy [23]. A proposition is $t$ true, $i$ indeterminate, and $f$ false, where $t, i$, and $f$ are real values from the ranges $T, I, F$ with no restriction on $T, I, F$ or the sum $n=t+i+f$.

Compared with all other logics, neutrosophic logic introduces a percentage of "indeterminacy" - due to the unexpected parameters hidden in some propositions. It also allows each component $t, i, f$ to "boil over" 100 or "freeze" under 0. Also neutrosophic logic is the generalised logic with would exhibit fuzzy logic properties under certain conditions, like when the sets are reduced to an element only respectively, then

$\mathrm{t} \_\sup =\mathrm{t} \_$inf $=\mathrm{t}, \mathrm{i} \_$sup $=\mathrm{i} \_$inf $=\mathrm{i}, \mathrm{f} \_$sup $=\mathrm{f}$ _inf $=\mathrm{f}$,

and $\mathrm{n} \_$sup $=\mathrm{n} \_$inf $=\mathrm{n}=\mathrm{t}+\mathrm{i}+\mathrm{f}$

Hence, the neutrosophic logic generalizes:

- the fuzzy logic (for $n=1$ and $i=0$, and $0 \leq t, i, f \leq 1$ )

So, as discussed above and in table I, neutrosophic logic is an answer to the current limitations of fuzzy inference systems and is an apt choice to be used by the futuristic inference systems.

\section{APPLICATION OF NEUTROSOPHIC LOGIC TO MEDICAL DOMAIN}

\subsection{Why neutrosophication of medical data - is useful}

Conventional AI is discrete in nature. AI simulation models are constructed from huge sets of symbols. As the complexity of the models constructed is directly proportional to the number of symbols employed, so the two properties of resolution and simplicity of AI models are mutually exclusive in nature. Thus, medical AI systems generally misses the transition which is essential for maintaining continuity of medical problem.

Like for example, it is expected that different patients which exhibit comparable symptoms be given analogous diagnoses.
But in reality diagnostic systems lacks this desirable essential property: this is possible because of system-specific thresholds, thus equivalent cases may be detached during the qualitative generalization of continuous parameters and are consequently treated differently, thus resulting in significantly differing diagnoses. Though it is expected that slight variations in the parameters values lead to slight change in the diagnosis, however, symbolic dy namic systems exhibits abrupt changes in the state for gradual transition in the parameter values.

To tackle the issue of introducing continuity in the deductive thinking fuzzy sets have been researched extensively and employed in the medical inference systems [1],[2],[3],[4],[5],[6],[7],[8],[9],[10],[11],[12],[13],,[14],[15],[16] . Fuzzy sets thus impart the gradual transition behaviour from one state to other state and permits traditional symbolic systems to display continuous behaviour. Fuzzy logic plays an important role in capturing the borderline cases as it permits gradual shift from one class to other class; as it decides the degrees of fit of what is actually present and its internal descriptions and proliferate these gradation through the system to its output, where they serve to qualify the results of the reasoning process. However for clear cut cases it exhibits same behavior similar to that of underly ing symbolic structure. For example, as the actual blood pressure of a patient increases, the degree of fit of the description 'low blood pressure' (or the degree of truth of an equivalent statement) decreases, and the degrees of truth of all conclusions derived there from change in the direction determined by the involved logical connectives. However fuzzy logic representation has its own limitations.

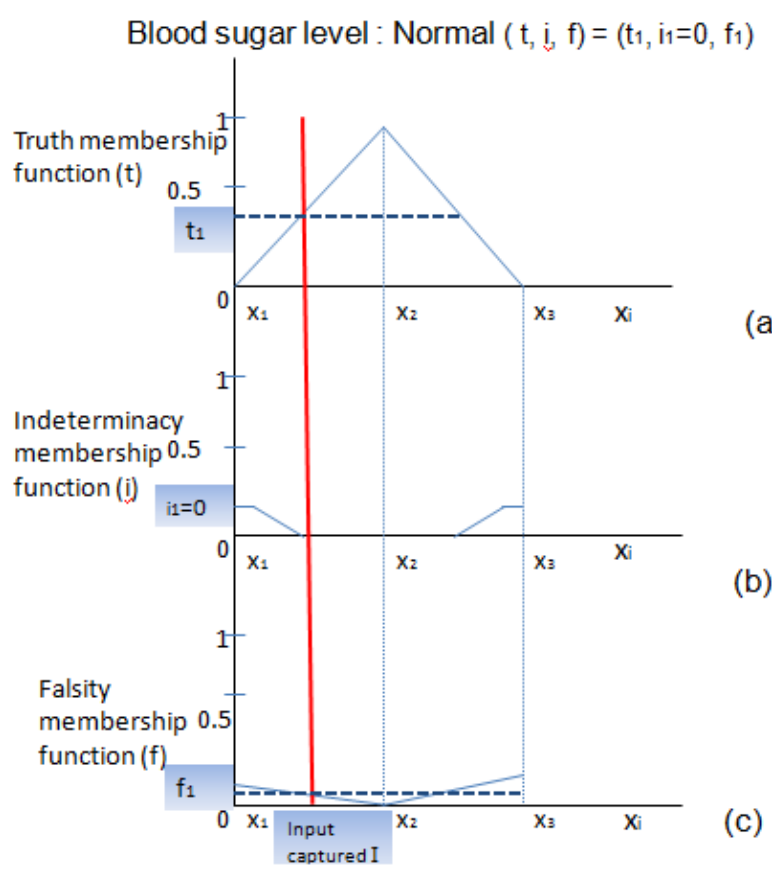

Figure 1: Neutrosophication of the input captured

As shown in the figure 1 neutrosophic logic would help in explicitly listing out three important components of the input values captured: truthness, indeterminacy and falsity. Figure 1 shows the conceptual membership functions of blood sugar 
level= normal; designed for capturing truthness, indeterminacy and falsity of the input recorded. The designing of these three membership functions would vary considerably for different parameters. Here in this figure, the captured input $(I)$ is mapped onto the three membership functions, here it is assumed that indeterminacy related to deciding whether the blood sugar is normal is high on the tapering ends of the truth membership functions designed and falsity membership function corresponds to the lab equipment error or degradation of equipment noticed which can give erroneous results. So as per the figure 1, the three component values generated after neutrosophication of captured input $I$ is $I\left(t_{l}, i_{l}=0, f_{l}\right)$.

Figure 2, discusses the neutrosophication process applied to multiple parameters simultaneously. As it is very common in medical domain to infer $D$, analysis of multiple parameters is required. Here in this figure it is assumed that analysis of 4 input parameters is required to infer $D$. As clearly shown input parameter $\left(I_{l}\right)$ lies in the indeterminacy zone (which could be possible due to lack of information or early onset of the disease during which input $I_{l}$ cannot be captured); input parameters $\left(I_{2}\right.$ and $I_{3}$ ) lies in the truth zone indicating favor for $\mathrm{D}$ and corresponding mapping shows $I_{3}$ favors strongly for $D$ in comparison to $I_{2}$ as $t_{1}>t_{2}$; input parameter $I_{4}$ contradicts the possibility of $D$ by $f_{l}$ value.

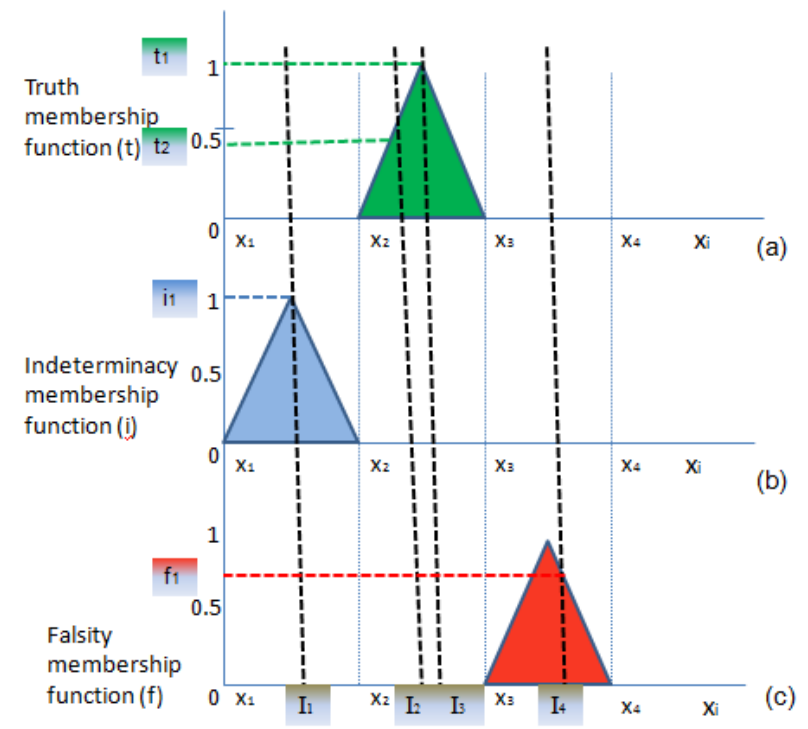

Figure 2: Neutrosophication involving multiple parameters

Fuzzy logic designs only the truth membership functions that gives description of degree of membership value to a particular class. Contrary to fuzzy logic in which there is no provision of capturing indeterminacy corresponding to non availability of information, or falsity functions to record the imprecision or degradation of the equipment with which input is captured, neutrosophic logic is definitely a better representation of the medical data as it gives the clear insight of the truthness, indeterminacy and falsity associated with the input captured.

This should indeed be of considerable interest to the medical AI community, because, as indicated above, medicine is essentially a continuous domain where the captured inputs could have uncertainty, indeterminacy and sometimes falsity associated.
Incorporation of neutrosophic logic in the medical models would retain the continuous behaviour as displayed by the fuzzy logic; such systems would essentially reduce to medical systems with fuzzy logic under special circumstances as discussed in section 2. Medical domain is the field where there is indeterminacy, unknown, hidden parameters, imprecision, high conflict between sources of information, non-exhaustive or non-exclusive elements of the frame of discernment so neutrosophy could be applied.

Similar to fuzzy systems, depending on the design of rule base of neutrosophic medical model, the output of such a system can be a continuous function (Sugeno model [24]) or it can be a single value output (Mamdani and Tsukamoto model [25], [26]). Generally the continuous output function would be a better estimation of the modelled medical relationship than its underlying discrete specification.

As suggested neutrosophic medical systems can be utilized for neutrosophic scores; continuous truth/indeterminate/falsity versions of conventional score schemes. The approach of incorporation of neutrosophic sets in medical domain would lead to tabular or rule-based mapping from input to output variables effectively implementing a continuous control law. Neutrosophic qualitative simulation and, more generally, neutrosophic model based diagnosis are promising candidates for future research.

The proposed neutrosophic logic theory is not a substitute of existing fuzzy medical models, but an extension and enhancement of classical AI approaches. Due to inherit advantages of neutrosophic sets; as discusses in section 2, such systems would address medical problems more adequately. What makes inclusion of neutrosophic logic in the medical domain a powerful tool is its desirable properties of allowing continuity, gradation of reality, capturing of truthness, indeterminacy and falsity.

\subsection{Currently neutrosophication is unusual but this unusualness is appropriate for medical data processing}

Contrary to the continuous and indeterminate nature of medicine domain; fuzzy systems though ensures continuity but are limited to handle complete information. So here arises contradiction that when values of blood pressure, heart rate or blood gases are acquired or obtained using mechanical equipments which have predefined imprecision factor and degradation associated; also many times required parameter value for a diagnosis is not available due to lack of knowledge or missing lab results then why should we only rely on fuzzy reasoning process which cannot handle the aforesaid issues. They deal with the membership degree to the fuzzy input/output classes and there is no provision of capturing the indeterminacy or falsity of the input acquired.

Rule bases of current medical fuzzy systems maps graded inputs to graded outputs, final value inferred after defuzzification. Using fuzzy logic to express medicine data is a viable option as it permits usage of numeric and textual information. There is no standard universally applicable to encode medical knowledge, it can contain numeric and textual information, different symbols can be used with same meaning, redundancy is possible, probability of erroneous misspell medical terms is also high, so 
representation technique used should be able to handle all these issues. As medical knowledge is ever changing so it is imperative that it is represented in qualitative and semiquantitative (fluid) manner. Medical information can be imprecise, partial, and vague or imperfect as some lab results may be missing from the feature set or due to patient's inability to answer properly. The performance of neutrosophic systems is likely to be closer to medical reality than that of their fuzzy counterparts. Neutrosophic sets holds the potential of bridging the gap the currently employed medical fuzzy systems and futuristic medical systems which would be able to deal with three important components of the data acquired and information generated; truth, indeterminacy and falsity.

The neutrosophic logic is a formal frame trying to measure the truth, indeterminacy, and falsehood.

a. Possibility of integrating neutrosophic logic with medical knowledge base is reasoned on the grounds that while opting for any particular therapeutic plan, any doctor would be -performing information fusion as the data would be coming from multiple sources like patients description of symptoms, laboratory data etc., so to model such systems it is essential that we should consider not only the truth -membership supported by the evidence but also the falsity membership against the evidence.

For example, when we ask the opinion of an expert doctor about certain statement, he or she may say that the possibility that the statement is true is between 0.5 and 0.7 , and the statement is false is between 0.2 and 0.4 , and the degree that he or she is not sure is between 0.1 and 0.3 .

Also neutrosophic logic allows time dependent values. For example values of 10 parameters is decisive for any diagnosis $D$, idealistic value would be $D(1,0,0)$ : which indicates that all the ten parameters are favouring for diagnosis $D$; but this rarely happens. Suppose at any time $t_{l}$, input values taken for 10 parameters resulted in $D(0.3,0.5,0.2)$; indicating three vote "yes", two vote "no" and five are undecided, at any time $t_{2}$, where $t_{2}>t_{l}$, neutrosophic value generated for diagnosis $D$ is $D$ $(0.6,0.2,0.2)$ indicating six vote "yes", two vote "no", two give up and two are undecided. So comparing two different values of $D$ captured at two different times clearly shows that as time progresses more and more parameter values are indicating for diagnosis $D$; which could be due to attainment of minimum threshold values by individual parameter value (in time $t_{2}-\mathrm{t}_{1}$ ) essential for pointing towards diagnosis $D$.

Similar to time dependent values, neutrosophic logic also allows observer dependent values: which again is in place with the complexities of medical domain. It is very common that the support for diagnosis $D$ would vary from one doctor to other depending on the individual expertise and judgment.

Fuzzy sets do not allow the provision of time dependent or observer dependent changes in membership values.

b. In neutrosophic set, indeterminacy is quantified explicitly and truth-membership, indeterminacy-membership and falsity-membership are independent. This assumption is very important in a lot of situations such as information fusion when we try to combine the data from different sensors.

c. Also as fuzzy logic is limited with the restriction that $t+f=$ 1 , so it is not capable to handle incomplete, indeterminate and inconsistent information which is very natural for any medical information. And neutrosophy by it's virtue of handling inconsistent and incomplete information seems to be a better choice for modeling medical knowledge base, because it is often not possible to have $100 \%$ of information at hand while making decision.

d. Neutrosophic Logic allows for the provision of absolute/relative truth and falsehood. If the neutrosophic truth-value of a statement $\mathrm{A}, \operatorname{NLt}(\mathrm{A})=1+$, if $\mathrm{A}$ is 'true in all possible worlds' and all conjunctures, that one may call "absolute truth", whereas $\operatorname{NLt}(\mathrm{A})=1$ if A is true in at least one world at some conjuncture, we call this "relative truth" because it is related to a 'specific' world and a specific conjuncture. Similarly for the neutrosophic falsehood-value, $\operatorname{NLf}(\mathrm{A})=1+$ if the statement $\mathrm{A}$ is false in all possible worlds, we call it "absolute falsehood", whereas $\operatorname{NLf}(A)=1$ if the statement $\mathrm{A}$ is false in at least one world, we call it "relative falsehood". Also, the neutrosophic indeterminacy value $\operatorname{NLi}(\mathrm{A})=1+$ if the statement $\mathrm{A}$ is indeterminate in all possible worlds, we call it "absolute indeterminacy", whereas $\operatorname{NLi}(A)=1$ if the statement $A$ is indeterminate in at least one world, we call it "relative indeterminacy". On the other hand, $\operatorname{NLt}(\mathrm{A})=-0$ if $\mathrm{A}$ is false in all possible world, whereas $\operatorname{NLt}(\mathrm{A})=0$ if $\mathrm{A}$ is false in at least one world; $\operatorname{NLf}(A)=-0$ if $A$ is true in all possible world, whereas $\operatorname{NLf}(A)=0$ if $A$ is true in at least one world; and $N \operatorname{Li}(A)=-$ 0 if $\mathrm{A}$ is indeterminate in no possible world, whereas $\operatorname{NLi}(\mathrm{A})=0$ if $\mathrm{A}$ is not indeterminate in at least one world. The -0 and $1+$ monads leave room for degrees of super-truth (truth whose values are greater than 1), super-falsehood, and super-indeterminacy.

\section{FUTURE WORK AND CONCLUSION}

It is quite clear that whatever inference systems are utilized be it Fuzzy inference systems (FIS) or neutrosophic inference system (NIS), the intention at this stage is not to replace or to substitute for an experienced doctor or detailed laboratory investigations; rather it is argued that FIS/NIS should be viewed as a decision aid for the busy expert (emergency expert); particularly in times of crisis, also these systems can also be augmented for raising alarm for any deviations monitored.

A novel theoretical and practical approach of utilizing neutrosophic logic in medical models justifies investigation now. Based on the concept of 'neutrosophic sets', it put forwards an alternative decision-making path that will permit us (and machines) to consider complex character of biological phenomena and thereby meet real clinical and experimental needs.

Though a trade off needs to be done between what is important currently: novel and more accurate sources of acquiring data or developing new reasoning techniques to deal with the available data; but certainly second option seems more viable when imprecision, uncertainty and gaps are integrated in the medical data acquired. 


\section{REFERENCES}

[1] L.A. Zadeh, Biological application of the theory of fuzzy sets and systems, in: Proc. Int. Symp. Biocybernetics of the Central Nervous System (Little, Brown \& Co., Boston, 1969) 199-212.

[2] Kalmanson D, Stegall HF. Cardiovascular investigations and

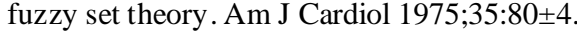

[3] A. O. Esogbue and R. C. Elder, "Fuzzy sets and the modeling of physician decision processes, part (II): Fuzzy diagnosis decision models," Fuzzy Sets Syst., vol. 3, no. 1, pp. $1-9,1980$.

[4] Adlassnig KP. A survey on medical diagnosis and fuzzy subsets. In: Gupta MM, Sanchez E, editors.Approximate reasoning in decision analy sis. Amsterdam: North-Holland, 1982. pp. $203 \pm 217$.

[5] C. Hughes. The representation of uncertainty in medical expert systems. Medical Informatics, 14:269-279, 1989.

[6] Maiers JE. Fuzzy set theory and medicine: the ®rst twenty years and beyond. In: Proceedings of the $9^{\text {th }}$ Symposium on Computer Applications in Medical Care. IEEE, 1985. pp. $325 \pm 329$.

[7] J.F. Martin, Editorial: Fuzzy control in anaesthesia, J. Clin. Monit. 10 (1994) 77-80.

[8] J. Wainer and S. Sandri, "Fuzzy temporal/categorical information in diagnosis," J. Intell. Inf. Syst., vol. 13, no. 1-2, pp. 9-26, 1996.

[9] K. Becker, B. Thull, H. Ka“smacher-Leidinger, J. Stemmer, G. Rau, G. Kalff and H.-J. Zimmermann, Design and validation of an intelligent patient monitoring and alarm system based on a fuzzy logic process model, Artif. Intell. Med. 11 (1997) 33-53.

[10] J.F. Hurdle, Leightweight fuzzy processes in clinical computing, Artif. Intell. Med. 11 (1997) 55-73.

[11] S.E. Kern, J.O. Johnson, D.R. Westenskow, Fuzzy logic for model adaptation of a pharmacokinetic- based closed loop delivery system for pancuronium, Artif. Intell. Med. 11 (1997) 9-31.

[12] F. Steimann, "Fuzzy set theory in medicine," Artif. Intell. Med., vol. 11, no. 1-7, 1997.
[13] J. Gamper and W. Nejdl, "Abstract temporal diagnosis in medical domains," Artif. Intell. Med., vol. 10, pp. 209-234, 1997.

[14] B. Kovalerchuk, E. Triantaphyllou, J.F. Ruiz, J. Clayton, Fuzzy logic in computer-aided breast cancer diagnosis: analy sis of lobulation, Artif. Intell. Med. 11 (1997) 75-85.

[15] K.-P. Adlassnig, "A fuzzy logical model of computer assisted medical diagnosis," Meth. Inf. Med., vol. 19, pp. 141-148, 1998.

[16] P. R. Innocent and R. I. John, "A fuzzy symptoms and a decision support index for the early diagnosis of confusable diseases," in Proc. RASC Conf.. Leicester, U.K., Jul. 2000.

[17] L. A. Zadeh, Fuzzy sets, Inf. Control 8 (1965), 338- 353.

[18] Klir, George J.; Yuan, Bo (1995). Fuzzy sets and fuzzy logic: theory and applications. Upper Saddle River, NJ: Prentice Hall PTR.

[19] Novák, Vilém (1989). Fuzzy Sets and Their Applications. Bristol: Adam Hilger.

[20] Van Pelt, Miles (2008). Fuzzy Logic Applied to Daily Life. Seattle, WA: No No No No Press.

[21] Zimmermann, H. (2001). Fuzzy set theory and its applications. Boston: Kluwer Academic Publishers.

[22] F. Smarandache (1999), Linguistic Paradoxists and Tautologies, Libertas Mathematica, University of Texas fat Arlington, Vol. XIX, 143-154.

[23] F.Smarandache (1999), A Unifying Field in Logics. Neutrosophy: Neutrosophic Probability, Set and Logic. Rehoboth: American Research Press.

[24] T. Takagi, M. Sugeno. "Fuzzy Identification of Systems and Its Applications to Modeling and Control", IEEE Transaction Systems, Man and Cybernetics, 15(1), pp.116132,1985

[25] E.H. Mamdami, S. Assilina, "An experiment in linguistic synthesis with a fuzzy logic controller", International Journal of Man-Machine Studies, vol. 7(1), pp. 1-13, 1975.

[26] Y. Tsukamoto, An Approach to Fuzzy Reasoning Method, Gupta M.M. et al (Eds.), Advances in Fuzzy Set Theory and Applications, pp. 137-149, 1979. 